\title{
Incidence of C-shaped root canal systems in mandibular second molars in the native Chinese population by analysis of clinical methods
}

\author{
Yan Wang, Jing Guo, Hai-Bing Yang, Xuan Han and Ying Yu \\ The aims of the study were to investigate the incidence of $\mathrm{C}$-shaped root canal systems in mandibular second molars in a native Chinese \\ population using radiography and clinical examination under microscope and to compare the relative efficacies of these methods. For \\ the recognition of C-shaped root canal system, 1146 mandibular second molars were selected and examined. Teeth with C-shaped \\ canal systems were categorized by using the radiographic classification criteria and the modified Melton's method. C-shaped canals \\ were identified in 397 (34.64\%) mandibular second molars by radiography (type I, 31.23\%; type II, 38.29\%; type III, 30.48\%). \\ Clinical examination showed that 449 (39.18\%) cases exhibited C-shaped canal systems (C1, 22.94\%; C2, 48.11\%; C3a, 15.59\%; \\ C3b, 13.36\%). As for the result of the radiographic and clinical combined examination, C-shaped root canals were found in 473 \\ $(41.27 \%)$ mandibular second molars (C1, 21.78\%; $C 2,45.67 \%$; $C 3 a, 16.70 \%$; $C 3 b, 15.86 \%)$. The incidence of $C$-shaped root canal \\ diagnosed by radiographic method was statistically different from that by clinical examination and the combined examination \\ $(P<0.05)$. The study indicated a high incidence of $\mathrm{C}$-shaped canal system in a Chinese population. The combination of microscopic \\ and radiographic examination is an effective method in identifying the $C$-shaped root canal system.
} International Journal of Oral Science (2012) 4, 161-165; doi:10.1038/ijos.2012.42; published online 27 July 2012

Keywords: C-shaped root canal; incidence; mandibular second molar

\section{INTRODUCTION}

The C-shaped canal system may be observed in maxillary molars, maxillary and mandibular premolars, but most commonly detected in mandibular second molars. ${ }^{1-7}$ Its basic anatomical feature is the presence of a fin or web connecting the individual root canals. ${ }^{5,8-9}$ Its formation occurred in the course of development. ${ }^{10}$ The variation of C-shaped root canal system appears to be genetically determined, and it has some relationship with ethnic origin. ${ }^{9}$ It could be found throughout the world, but the incidence is much higher in Asian population. ${ }^{8,10-14}$ The incidence of C-shaped canal systems in extracted teeth for the Chinese population has been reported from $31.5 \%$ to $42 \%{ }^{15-17}$

The variations of the root canal anatomy along its root length present major challenges with respect to thorough debridement and proper obturation. ${ }^{9,18}$ Thus, correct diagnosis and identification of the C-shaped root canal before treatment is an essential step. The radiographic examination is a convenient, effective and non-invasive method to show the morphology of the root canal system. The operative microscopic examination is capable of providing distinct vision field, rendering it possible to observe the morphologic characteristics of the pulp chamber floor and orifice directly and facilitating the recognition of C-shaped root canal system.

The purpose of this study was to investigate the incidence of Cshaped root canal systems in mandibular second molars in a native
Chinese population using radiography and clinical examination under microscope and compare the relative efficacies of these methods, in the hope of providing a reliable guidance for endodontic treatment and enhancing its success rate.

\section{MATERIAL AND METHODS}

All subjects were the patients in the Department of Endodontics, School and Hospital of Stomatology, Shandong University, China from 2004 to 2009. Their consents to participate in this study were ensured before the study and the protocols were approved by the Medical Ethics Committee of Shandong University. Basic information of the subjects was recorded with respects to their ethnicity, gender, age and history of past treatment. In this study, 1146 mandibular second molars scheduled for root canal therapy were randomly selected. Among these teeth, 609 were males and 537 were females, with the age ranging from 23 to 78 years. In order to make sure that the subjects were purebred Chinese, they were selected only from indigenous Chinese patients. All teeth were evaluated by radiographic and clinical examination, respectively.

Radiographic examination and its identification categories In the radiographic examination, two preoperative radiographs, one working length radiograph and two postoperative radiographs were

Department of Endodontics, School of Stomatology, Shandong University, Shandong Provincial Key Laboratory of Oral Biomedicine, Jinan, China

Correspondence: Dr Y Wang, Department of Endodontics, School and Hospital of Stomatology, Shandong University, 44-1 Wenhua Xi Road, Jinan 250012, China

E-mail: wangyan1965@sdu.edu.cn

Received 15 January 2012; revised 16 May 2012 


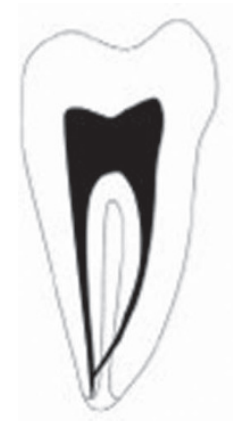

a

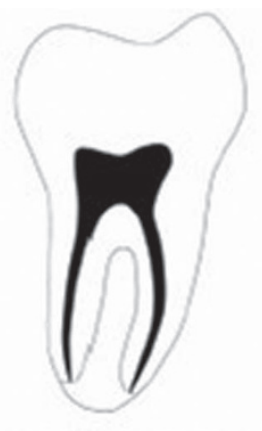

b

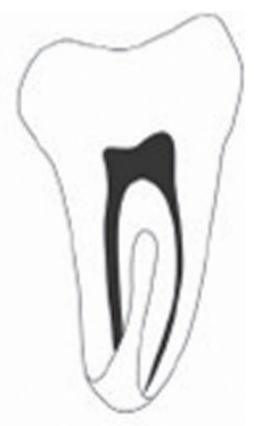

C
Figure 1 Radiographic types. (a) Type I: a mesial and a distal canal merge into one before exiting at the apical foramen. (b) Type II: there are a mesial and a distal canal; the two canals appeared to continue on their own pathways to the apex. (c) Type III: there are a mesial and a distal canal; one canal curves to and superimposes on the radiolucent line when running towards the apex, and the other canal appears to continue on its own pathway to the apex.
C1

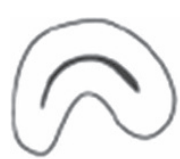

C3a

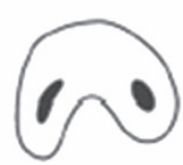

C2

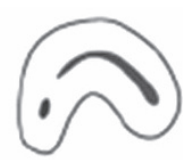

C3b

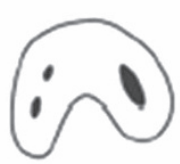

C4

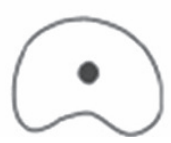

Figure 2 Classification of the canal configuration of the modified Melton's method. Category C1: the shape is an uninterrupted ' $C$ ' with no separation or division. Category C2: the canal shape resembles a semicolon resulting from a discontinuation of the ' $\mathrm{C}$ ' outline. Category C3a: two separated canals. Category C3b: three separated canals. Category C4: only one round or oval canal.

taken for each case. One of the preoperative radiographs was with a $90^{\circ}$ angulation to the tooth in a bucco-lingual direction and the other was at a mesial angle of approximately $20^{\circ}$ to make the radiographs better visualized of the bucco-lingual anatomy. Two postoperative radiographs were taken by the same method as the preoperative ones to confirm canal configuration. The radiographs were exposed with a digital dental X-ray system (Sirona, Bensheim,
Germany) and analyzed by three experienced endodontic dentists individually. If there was any disagreement among them, a radiologist with endodontic experience helped to make the final decision.

The C-shaped canals were identified and classified in accordance with their radiographic appearances proposed by researchers ${ }^{5,9,18}$ (Figure 1).

\section{Clinical examination and its identification categories}

Clinical examination was carried out with the help of an operative microscope (OPMI Movena, Zeiss Company, Oberkochen, Germany). At $\times 10$ magnification, careful probing of pulp chamber floor and canal entrance with a small file following the access to pulp chamber was performed. A radiograph with no. 10 or no. $8 \mathrm{~K}$-file in the canal was taken aiming to determine the work length and demonstrates root canal configuration. After root canal obturation was finished, another two post-radiographs with the same method as the preoperative were taken to confirm the canal morphology.

The modified Melton's method proposed by researchers ${ }^{5,9,19-20}$ was used to identify and classify the C-shaped root canal from clinical examination (Figure 2).

\section{Statistic analysis}

The incidence of C-shaped root canals examined by the methods of radiographic appearance, clinical examination and the combined methods were calculated, respectively. The data were subjected to the Chi-squared test at a significance level of 0.05 . All statistical analyses were performed using SPSS 17.0 software for Windows.

\section{RESULTS}

According to radiographic characteristics, of 1146 cases, the incidence of C-shaped canal systems was 34.64\%. As for the C-shaped root canal, type I, type II and type III accounted for $31.23 \%, 38.29 \%$ and $30.48 \%$, respectively. The X-ray appearances are presented in Figure 3. Based on the pulp chamber floor and orifice configuration observed under microscope, our group detected that 449 (39.18\%) presented Cshaped canal systems. According to the modifications of Melton's classification method, among the teeth with C-shaped root canal systems, 103 (22.94\%) presented category C1, 216 (48.11\%) presented category C2, $70(15.59 \%)$ and $60(13.36 \%)$ presented category C3a and $\mathrm{C} 3 \mathrm{~b}$, respectively. 473 were identified to be C-shaped root canals via the combined method, with the incidence at $41.27 \%$. Among C-shaped root canal systems recognized, 103(21.78\%) exhibited category C1, $216(45.67 \%)$ presented category C2, $79(16.70 \%)$ and 75 (15.86\%) exhibited category $\mathrm{C} 3 \mathrm{a}$ and $\mathrm{C} 3 \mathrm{~b}$, respectively. The classification of the identified $\mathrm{C}$-shaped root canal and their radiographic appearances are shown in Figure 4. The percentage of all categories in identified C-shaped root canal systems by different methods is shown in Table 1.

The statistic analysis showed that there were significant differences between the groups by using radiographic and clinical examination
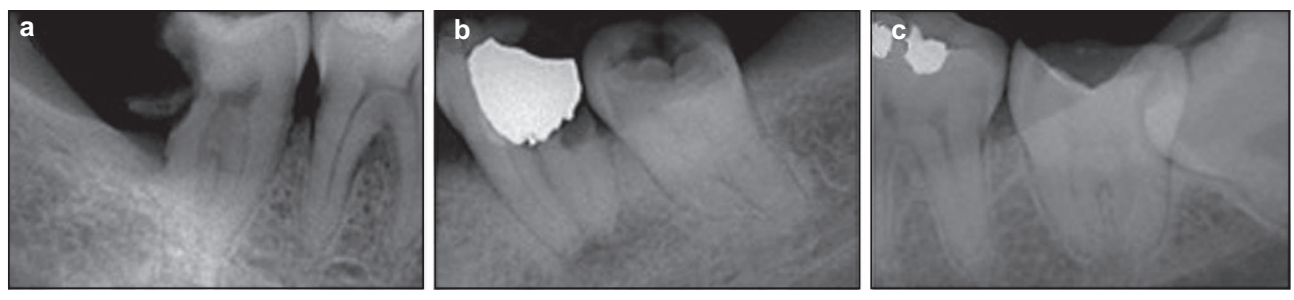

Figure 3 Radiographic classification based on preoperative X-ray. (a) Type I; (b) type II; (c) type III. 

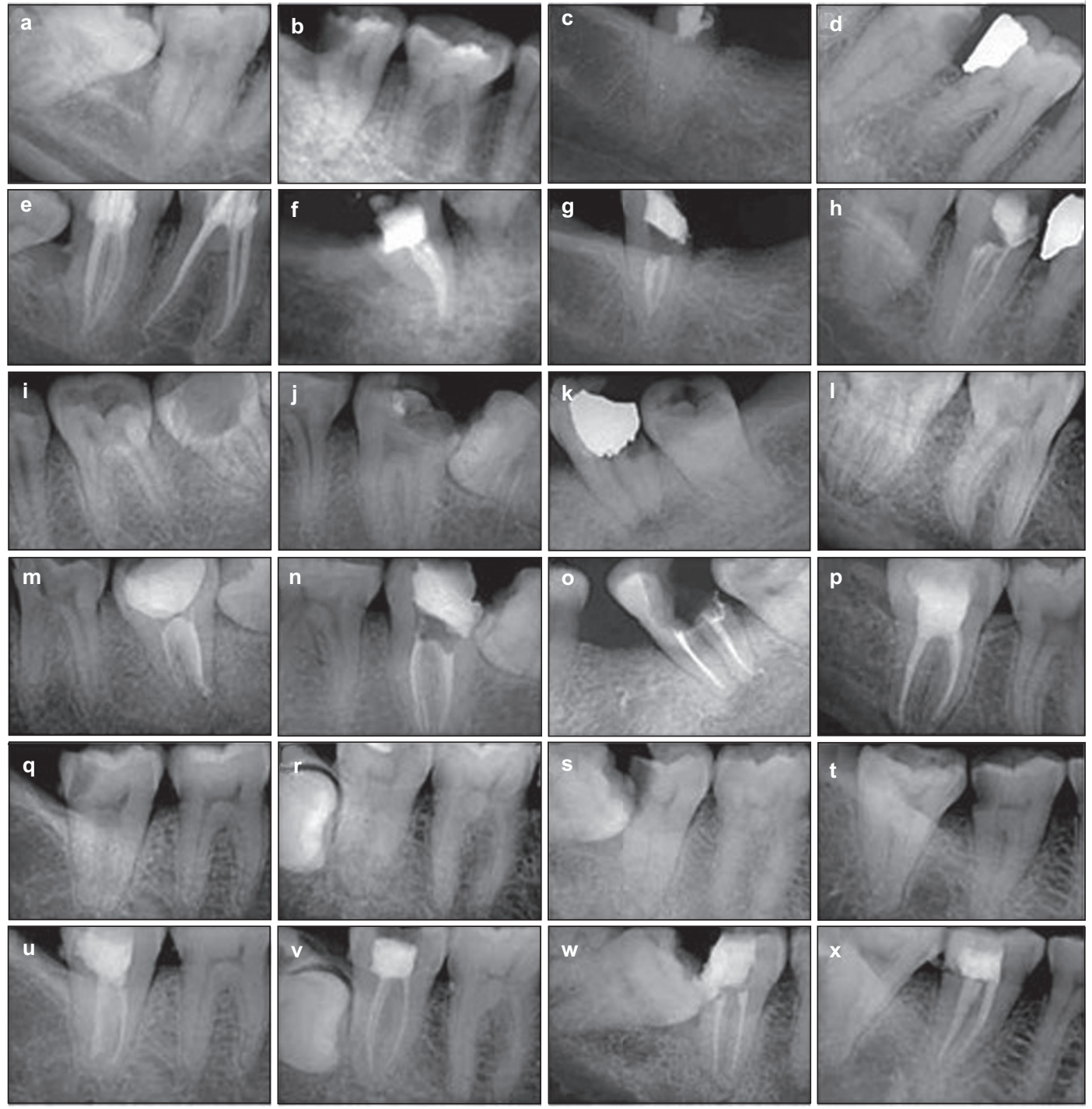

Figure 4 The $\mathbf{X}$-rays of preoperation and postoperation. Type I: (a-d) the X-rays of preoperation; (e-h) the X-rays of postoperation, they belonged to $\mathrm{C} 1$, $\mathrm{C} 2$, $\mathrm{C} 3 \mathrm{a}$ and C3b. Type II: (i-I) the X-rays of preoperation; $(\mathbf{m}-\mathbf{p})$ the X-rays of postoperation, they belonged to C1, C2, C3a and C3b. Type III: (q-t) the X-rays of preoperation; $(\mathbf{u}-\mathbf{x})$ the X-rays of postoperation, they belonged to C1, C2, C3a and C3b.

Table 1 Number and percentage of all categories in identified Cshaped root canal systems by different methods

\begin{tabular}{lc}
\hline Methods & $\begin{array}{c}\text { Number of teeth with C-shaped canal systems } \\
\text { of different category (percentage) }\end{array}$ \\
\hline Radiographic examination & $397(100 \%)$ \\
Type I & $124(31.23 \%)$ \\
Type II & $152(38.29 \%)$ \\
Type III & $121(30.48 \%)$ \\
Clinical examination & $449(100 \%)$ \\
C1 & $103(22.94 \%)$ \\
C2 & $216(48.11 \%)$ \\
C3a & $70(15.59 \%)$ \\
C3b & $60(13.36 \%)$ \\
Combined methods & $473(100 \%)$ \\
C1 & $103(21.78 \%)$ \\
C2 & $216(45.67 \%)$ \\
C3a & $79(16.70 \%)$ \\
C3b & $75(15.85 \%)$ \\
\hline
\end{tabular}

$(P<0.05)$, as well as using radiographic and combined examination $(P<0.05)$, whereas the groups of clinical and combined examination showed no statistic difference $(P>0.05)$.

\section{DISCUSSION}

Incidence of C-shaped root canal systems in mandibular second molars and the related key factors

In this study, the incidence of C-shaped root canal systems in a Chinese population (41.27\%) was higher than that in Yang's study $(31.5 \%) .{ }^{15}$ Several possible reasons may account for the different incidence in the same ethnic population. Firstly, the difference of the incidence may due to the disparity of the experimental teeth. The subjects of Yang et al. were extracted teeth which may fail to represent those in the natural population. In contrast, the teeth in this study were still the living ones scheduled for root canal treatment, which would function better to represent the natural population. Secondly, the samples in this study were much more than that of 
Yang et al. Thirdly, the clearing technique in the study of Yang et al. showed the anatomic configuration in three dimensions better than radiograph and clinic examination in our study, but this technique cannot be used in clinic practice. Finally, the criteria of diagnosis and classification in our study were different from those of Yang et al. We assumed more novel and detailed criteria of diagnosis and classification, ${ }^{5}$ compared to the relatively simple ones in Yang's study. In another report, the incidence of fused roots in mandibular second molars of ethnic Chinese in Hong Kong based on radiograph was reported as $42 \% .{ }^{16}$ Considering the possibility that a small proportion of the fused root teeth may lack a C-shaped root canal system, the real incidence of C-shaped root canal systems is likely to be lower than $42 \%$. A recent study reported that a C-shaped canal system was present in $39 \%$ of mandibular second molars in a Chinese population evaluated by cone-beam computed tomography. ${ }^{17}$ The incidence of $\mathrm{C}$-shaped root canal systems in the above study was close to that in our study.

Cooke and Cox suggested that it is impossible to diagnose C-shaped canals based on pre-operative radiographs ${ }^{1}$ and other studies showed that only a small percentage of C-shaped mandibular second molars were recognized. ${ }^{3}$ However, based on the complete procedure of radiographic examination, 20 out of 22 mandibular second molars with C-shaped canals were identified in Lambrianidis' study. ${ }^{3}$ In this study, among the 1146 cases, 397 were diagnosed as C-shaped root canal by radiographs. Besides, 76 cases with two separate roots in preoperative radiographs were recognized as C-shaped root canal after clinical examination, among which 7, 11, 36 and 22 cases were classified as $\mathrm{C} 1, \mathrm{C} 2, \mathrm{C} 3 \mathrm{a}$ and $\mathrm{C} 3 \mathrm{~b}$, respectively. The reason was that the dentine fin was so thin that the image was invisible on the radiograph. Under such circumstances, probing into the pulp chamber and orifice is indispensable.

Four hundred and forty-nine mandibular second molars were identified in the clinical examination, whereas 473 were diagnosed as C-shaped root canal by radiograph and clinical combined examination. There was no statistic difference when comparing the incidence of C-shaped root canal detected through clinical examination with that using radiographic plus clinical examination $(P>0.05)$, which may be due to the insufficient number of subjects. Moreover, by clinical examination itself, 24 C-shaped root canal systems that belonged to C3a and C3b orifice were considered as separate canals thus being overlooked, but when referring to radiograph, they were normally identified.

\section{Configurations of the pulpal chamber floor and orifice, radiographic appearance and clinical significance}

This study demonstrated a larger apico-occlusal dimension with a more apically positioned furcation in teeth that had C-shaped canal systems. The pulp chamber floors of teeth with C-shaped root canal systems were not convex, but deep and more concave. Our group detected 103 (21.78\%) C-shaped root canal orifice (C1) without difficulty. Several studies have suggested that a C-shaped orifice is an important feature that helps identify C-shaped root canal systems. ${ }^{3,15,20}$ However, more attention should be paid to the non-Cshaped orifice because they are usually disregarded as a C-shaped root canal system. In this study, C2 and C3 the orifice accounted for most of the C-shaped root canal systems (370, 78.22\%). Although they might appear to be semicolon or separate orifice, an isthmus linking root canal is often visible. Meanwhile, we found that there were two kinds of C2 orifice: one included a mesio-buccal ribbon-shaped and a distal root canal and the other a disto-buccal ribbon-shaped and a mesio-lingual root canal. In contrast to the former, the mesio-lingual root canals were always narrow and curved. Chai and Thong ${ }^{21}$ reported that lingual walls were normally thinner than buccal walls at different levels along the $\mathrm{C}$-shaped root canal. All the above features rendered the treatment of C-shaped root canal systems more difficult. Practitioners should be aware of these factors to avoid perforation during the course of treatment.

Radiographs of most teeth with C-shaped root canals usually showed tapered or square fused root outline, while separate and adjacent root canals may appear. A radiolucent longitudinal groove separating the root into mesial and distal parts was observed. The presence of a file or filling materials in the canal could facilitate radiographic recognition. In 121 cases that were classified as radiographic type III, one canal appeared to continue along its pathway to the apex, while the other curved to and superimposed into the radiolucent line when running towards the apex. In this situation, special care should be taken so as to avoid overpreparation and destruction of apical structure.

Because the shape varied along the length of the root, the configuration characteristics of $\mathrm{C}$-shaped canal orifice observed under the operative microscope should not be considered as the same all through its length. Haddad et al. ${ }^{10}$ believed that specific radiographic characteristics could help predict the existence of C-shaped root canal systems. In this study, 397 (83.93\%) from 473 cases of C-shaped root canal systems were identified by preoperative radiograph. In our opinion, it was possible to predict the presence and the configuration of C-shaped canal system by the radiographic appearance. What's more, clinical examination under microscope would make the recognition more accurate.

\section{CONCLUSION}

Chinese native population exhibited a high incidence of C-shaped root canal systems of mandibular second molars. Radiographs in combination with clinical examination under microscope could provide an accurate recognition clinically. Thus, clinicians need to know the presence of $\mathrm{C}$-shaped root canal systems and their configuration characteristics in order to increase the success rate of endodontic treatment.

\section{ACKNOWLEDGMENTS}

This study was supported by the grant from the Independent Innovation Foundation of Shandong University of China (No. 2011JC019) and the grant of Science and Technique Development Foundation of Shandong province (2010G0020230).

1 Cooke HG, Cox FL. C-shaped canal configurations in mandibular molars. J Am Dent Assoc 1979; 99(5): 836-839.

2 Weine FS, Members of the Arizona Endodontic Association. The C-shaped mandibular second molar: incidence and other considerations. J Endod 1998; 24(5): 372-375.

3 Lambrianidis T, Lyroudia K, Pandelidou 0 et al. Evaluation of periapical radiographs in the recognition of C-shaped mandebular second molars. Int Endod J 2001; 34(6): $458-452$.

4 Jerome CE. C-shaped root canal system: diagnosis, treatment, and restoration. Gen Dent 1994; 42(5): 424-427.

5 Fan B, Cheung GS, Fan M et al. C-shaped canal system in mandibular second molars: part II-Radiographic features. J Endod 2004; 30(12): 904-908.

6 Kottoor J, Velmurugan N, Ballal S et al. Four-rooted maxillary first molar having C-shaped palatal root canal morphology evaluated using cone-beam computerized tomography: a case report. Oral Surg Oral Med Oral Radiol Endod 2011; 111(5). e41-e45.

7 Sandhya R, Velmurugan N, Kandaswamy D. Assessment of root canal morphology of mandibular first premolars in the Indian population using spiral computed tomography: an in vitro study. Indian J Dent Res 2010; 21(2): 169-173. 
8 Gulabivala K, Opasanon A, Ng YL et al. Root and canal morphology of Thai mandibular molars. Int Endod J 2002; 35(1): 56-62.

9 Fan B, Cheung GS, Fan M et al. C-shaped canal system in mandibular second molars: Part I-Anatomical features. J Endod 2004; 30(12): 899-903.

10 Haddad GY, Nehme WB, Ounsi HF. Diagnosis, classification, and frequency of C-shaped canals in mandibular second molars in the Lebanese population. J Endod 1999; 25(4): 268-271.

11 Al-Fouzan. C-shaped root canals in mandibular second molars in a Saudi Arabian population. Int Endod J 2002; 35(6): 499-504.

12 Seo MS, Park DS. C-shaped root canals of mandivular second molars in a Korean popuIation: clinical observation and in vitro analysis. Int Endod J 2004; 37(2): 139-144.

13 Jin GC, Lee SJ, Roh BD. Anatomical study of C-shaped canals in mandibular second molars by analysis of computed tomography. J Endod 2006; 32(1): 10-13.

14 Al-Qudah AA, Awawdeh LA. Root and canal morphology of mandibular first and second molar teeth in a Jordanian population. Int Endod J 2009; 42(9): 775-784.

15 Yang ZP, Yang SF, Lin YC et al. C-shaped root canals in mandibular second molars in a Chinese population. Endod Dent Traumatol 1988; 4(4): 160-163.

16 Cheung LH, D L, Cheung GS. Root morphology — a study of the mandibular second molar of ethnic Chinese. Ann R Australas Coll Dent Surg 2006; 18: 47-50.
17 Zheng Q, Zhang L, Zhou X et al. C-shaped root canal system in mandibular second molars in a Chinese population evaluated by cone-beam computed tomography. Int Endod J 2011; 44(9): 857-862.

18 Jafarzadeh $\mathrm{H}$, Wu YN. The C-shaped root canal configuration: a review. J Endod 2007; 33(5): 517-523.

19 Melton DC, Krell KV, Fuller MW. Anatomical and histological features of C-shaped canals in mandibular second molars. J Endod 1991; 17(8): 384-388.

20 Min Y, Fan B, Cheung GS et al. C-shaped canal system in mandibular second molars part III: The morphology of the pulp chamber floor. J Endod 2006; 32(12): 11551159.

21 Chai WL, Thong YL. Cross-sectional morphology and minimum canal wall widths in C-shaped roots of mandibular molars. J Endod 2004; 30(7): 509-512.

(c) This work is licensed under a Creative Commons Attribution-NonCommercial-NoDerivative Works 3.0 Unported License. To view a copy of this license, visit http:// creativecommons.org/licenses/by-nc-nd/3.0 